S. Reich-Schupke ${ }^{1}$ J. Alm ${ }^{2} \cdot$ P. Altmeyer ${ }^{1} \cdot$ D. Bachter ${ }^{3} \cdot$ C. Bayerl ${ }^{4}$ - S. Beissert ${ }^{5}$. T. Bieber ${ }^{6} \cdot$ J. Böhmer $^{7} \cdot$ D. Dill ${ }^{8} \cdot$ E. Dippel $^{9} \cdot$ P. Dücker ${ }^{10} \cdot$ I. Effendy ${ }^{11} \cdot$ S. El Gammal $^{12} \cdot$ P. Elsner ${ }^{13} \cdot$ A. Enk ${ }^{14} \cdot$ I. Feldmann-Böddeker ${ }^{15} \cdot$ H. Frank ${ }^{16}$. W. Gehring ${ }^{17} \cdot$ U. Gieler ${ }^{18} \cdot$ M. Goebeler ${ }^{19} \cdot$ T. Görge ${ }^{20} \cdot$ H. Gollnick ${ }^{21} \cdot$ S. Grabbe ${ }^{22}$.

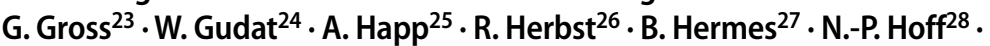
S.M. John ${ }^{29} \cdot$ M. Jungelhülsing ${ }^{30} \cdot$ M. Jünger ${ }^{31}$ - M. Kaatz ${ }^{32}$ - A. Kapp ${ }^{33}$. R. Kaufmann ${ }^{34}$. J. Klode ${ }^{35}$. K. Knaber ${ }^{36}$. A. König ${ }^{37}$. T. Krieg ${ }^{38}$. P. Kohl ${ }^{39}$. L. Kowalzick ${ }^{40} \cdot$ P. Lehmann ${ }^{41} \cdot$ H. Löffler ${ }^{42} \cdot$ J. Maschke ${ }^{43} \cdot$ W. Marsch $^{44}$. D. Mechtel ${ }^{45} \cdot$ P. Mohr ${ }^{46} \cdot$ I. Moll ${ }^{47} \cdot$ M. Müller48 $\cdot$ D. Nashan ${ }^{49} \cdot$ H.M. Ockenfels ${ }^{50}$. R.U. Peter ${ }^{51} \cdot$ H. Pillekamp ${ }^{52} \cdot$ R. Rompel ${ }^{53} \cdot$ T. Ruzicka $^{54} \cdot$ K. Salfeld ${ }^{55} \cdot$ C. Sander ${ }^{56}$. J. Schaller ${ }^{57} \cdot$ K. Scharffetter-Kochanek ${ }^{58} \cdot$ G. Schuler ${ }^{59} \cdot$ H.-J. Schulze ${ }^{60}$.

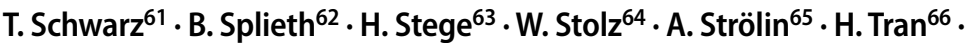
M. Tronnier ${ }^{67} \cdot$ J. Ulrich ${ }^{68} \cdot$ T. Vogt $^{69} \cdot$ G. Wagner ${ }^{70} \cdot$ J. Welzel $^{71} \cdot$ T. Willgeroth $^{72}$.

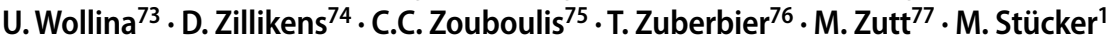

\title{
Phlebologie an deutschen Hautkliniken
}

\section{Eine Bestandsaufnahme im Auftrag der Deutschen Gesellschaft für Phlebologie}

Die Zahl der Dermatologen, die Mitglied in der Deutschen Gesellschaft für Phlebologie (DGP) sind, ist entsprechend den letzten Jahresberichten rückläufig. Fraglich sind die Ursachen für diese Entwicklung. Zur Bestandsaufnahme der Situation der Phlebologie an deutschen Hautkliniken wurde eine Befragungsstudie im Auftrag der DGP durchgeführt.

\section{Epidemiologie und sozioökonomische Bedeutung der Varikose}

Venenleiden gehören $\mathrm{zu}$ den großen Volkskrankheiten unserer Zeit. Etwa 20\% der Bevölkerung sind entsprechend der Bonner Venenstudie von einem Venenleiden betroffen [12]. Die Inzidenz und Schwere der Venenerkrankungen steigen mit dem Alter der Betroffenen. Angesichts der aktuellen demografischen Entwicklung mit einer Zunahme älterer Menschen ist von einem Anstieg an behandlungsbedürftigen Venenleiden auszuge- hen. Schon jetzt ist die sozioökonomische Bedeutung von Venenleiden enorm. Die entstehenden Kosten resultieren aus Untersuchung und Behandlung sowie einer Verschlechterung der Lebensqualität der Betroffenen und dem vorübergehenden oder gar vollständigen Verlust der Arbeitsfähigkeit durch das Venenleiden und seine Folgen. Verschärft wird das Problem durch den chronisch progredienten Verlauf und die Rezidivneigung der Varikose und der chronischen venösen Insuffizienz (CVI; $[4,10])$.

Entsprechend den Daten des statistischen Bundesamtes aus dem Jahr 2006 verursachten Varizenerkrankungen (ICD10 I83) ca. 2,18 Mrd. EUR Ausgaben pro Jahr [13]. Allein von 1980 bis 1990 hat es einen Kostenanstieg in Deutschland um 103\% für die CVI gegeben [5]. Stationäre Behandlungskosten hatten hier einen Anteil von etwa 230 Mio. EUR, ambulante Kosten von 234 Mio. EUR und Medikamente von 207 Mio. EUR [5].
Etwa $80 \%$ der durch Varizen entstehenden Kosten in der Bundesrepublik Deutschland entfielen 2006 auf die Gruppe der über 45-Jährigen [14]. Der Anteil der behandelten Frauen ist bei Varizenerkrankungen fast 3-mal so hoch wie bei den Männern (2,7:1; [13]). Allein das Ulcus cruris verursacht in Deutschland direkte und indirekte Jahrestherapiekosten von fast 10.000 EUR pro Patient [11].

Von großer Bedeutung sind außerdem die indirekten Kosten durch Arbeitsunfähigkeit jüngerer Erkrankter [9, 10]. Die meisten Krankschreibungen sind dabei auf operative Varizeneingriffe zurückzuführen [13]. Eine französische Studie konnte zeigen, dass etwa $7 \%$ der arbeitenden Bevölkerung wegen venöser Erkrankungen mit einer geschätzten Ausfallzeit von 4 Mio. Krankheitstagen in einem Jahr am Arbeitsplatz fehlen. Es ergeben sich

Die Autorenaffilationen finden Sie in der Infobox. 


\section{Infobox}

${ }^{1}$ Klinik für Dermatologie, Venerologie und Allergologie, Venenzentrum der Dermatologischen und Gefäßchirurgischen Kliniken, Ruhr-Universität Bochum; ${ }^{2}$ Dermatologikum, Hamburg; ${ }^{3}$ Klinik für Dermatologie, Venerologie und Allergologie, Carl-Thieme-Klinikum, Cottbus; ${ }^{4}$ Klinik für Dermatologie und Allergologie, Hauttumorzentrum, Wiesbaden; ${ }^{5}$ Klinik und Poliklinik für Dermatologie, Universitätsklinikium Carl-Gustav-Carus, Dresden; ${ }^{6}$ Klinik und Poliklinik für Dermatologie und Allergologie, Universitätsklinikum Bonn; ${ }^{7}$ Klinik für Dermatologie und Phlebologie, Katharinen-Hospital, Unna; ${ }^{8}$ Hautklinik, Märkische Kliniken, Lüdenscheid; ${ }^{9}$ Hautklink, Klinikum Ludwigshafen; ${ }^{10}$ Hautklinik, Allgemeines Krankenhaus Hagen; ${ }^{11}$ Hautklinik, Klinikum Bielefeld Rosenhöhe, Bielefeld; ${ }^{12}$ Hautklinik, Diakonie Klinikum Bethesda, Freudenberg; ${ }^{13}$ Klinik für Hautkrankheiten, Universitätsklinikum Jena; ${ }^{14}$ Hautklinik, Universitätsklinikum Heidelberg; ${ }^{15}$ Hautklinik, DRK-Krankenhaus, Chemnitz-Rabenstein; ${ }^{16}$ Fachklinik Sanaderm, Bad Mergentheim; ${ }^{17}$ Hautklinik, Städtisches Klinikum Karlsruhe; ${ }^{18}$ Klinik für Dermatologie, Venerologie und Allergologie, Universitätsklinikum Gießen; ${ }^{19}$ Klinik für Dermatologie, Venerologie und Allergologie, Universitätsklinikum Würzburg; ${ }^{20}$ Klinik für Hautkrankheiten, Universitätsklinikum, Münster; ${ }^{21}$ Klinik und Poliklinik für Dermatologie und Venerologie, Universität Magdeburg; ${ }^{22}$ Hautklinik und Poliklinik, Universitätsmedizin der Johannes Gutenberg-Universität Mainz ${ }^{23}$ Hautklinik, Universitätsklinikum Rostock; ${ }^{24}$ Silberg-Klinik, Bodenmais; ${ }^{25}$ Klinik für Dermatologie, Klinikum Frankfurt/Oder; ${ }^{26}$ Klinik für Dermatologie und Allergologie, Helios Klinikum Erfurt; ${ }^{27}$ Dermatologie \& Phlebologie, Vivantes-Klinkum im Friedrichshain, Berlin; ${ }^{28}$ Hautklinik, Universitätsklinikum, Düsseldorf; ${ }^{29}$ Dermatologisches Zentrum, Berufsgenossenschaftliches Unfallkrankenhaus, Hamburg; ${ }^{30}$ Klinik für Dermatologie, Venerologie und Allergologie, Ernst-von-Bergmann-Klinikum, Potsdam; ${ }^{31}$ Klinik und Poliklinik für Hautkrankheiten, Universitätsmedizin Greifswald; ${ }^{32}$ Klinik für Hautkrankheiten und Allergologie, SRW-Waldklinikum Gera; ${ }^{33}$ Klinik für Dermatologie, Allergologie und Venerologie, Medizinische Hochschule Hannover; ${ }^{34}$ Klinik für Dermatologie, Venerologie und Allergologie, Klinikum und Fachbereich Medizin, Johann Wolfgang Goethe-Universität, Frankfurt/Main; ${ }^{35}$ Klinik für Dermatologie, Universitätsklinikum Essen; ${ }^{36}$ Klinik für Dermatologie und Allergologie, Südharzklinikum, Nordhausen; ${ }^{37}$ Klinik für Dermatologie und Allergologie, Universitätsklinikum Marburg; ${ }^{38}$ Klinik und Poliklinik für Dermatologie und Venerologie, Uniklinik Köln; ${ }^{39}$ Klinik für Dermatologie \& Venerologie, Vivantes-Klinikum Neukölln, Berlin; ${ }^{40}$ Klinik für Hautkrankheiten und Allergologie, Helios Vogtland-Klinikum, Plauen; ${ }^{41}$ Klinik für Dermatologie, Allergologie und Dermatochirurgie, HeliosKlinikum, Wuppertal; ${ }^{42}$ Hautklinik, Klinikum am Gesundbrunnen, SLK-Kliniken, Heilbronn; ${ }^{43}$ Hautklinik, Städtisches Klinikum, Görlitz; ${ }^{44}$ Universitätsklinik und Poliklinik für Dermatologie und Venerologie, Universitätsklinikum Halle/Saale; ${ }^{45}$ Klinik für Hautkrankheiten und Allergologie, Heinrich-Braun-Klinikum, Zwickau; ${ }^{46}$ Dermatologisches Zentrum Buxtehude, Elbe-Kliniken, Buxtehude; ${ }^{47}$ Klinik und Poliklinik für Dermatologie und Venerologie, Universitätsklinikum Hamburg-Eppendorf, Hamburg; ${ }^{48}$ Universitätshautklinik, Universitätsklinikum Freiburg; ${ }^{49}$ Hautklinik, Klinikum Dortmund; ${ }^{50}$ Haut- und Allergieklinik, Klinikum Hanau; ${ }^{51}$ Hautklinik, Blausteinklinik, Blaustein; ${ }^{52}$ Klinik für Dermatologie und Venerologie, Bundeswehrkrankenhaus, Ulm; ${ }^{53}$ Klinik für Dermatologie, Klinikum Kassel; ${ }^{54}$ Klinik und Poliklinik für Dermatologie und Allergologie, LMU-München, München; ${ }^{55}$ Artemed Fachklinik, Bad Oeynhausen; ${ }^{56}$ Klinik für Dermatologie und Allergologie, Asklepios Klinik St. Georg, Hamburg; ${ }^{57}$ Klinik für Dermatologie, Allergologie und Umweltmedizin, Helios St. Barbara Klinik, Duisburg; ${ }^{58}$ Klinik für Dermatologie und Allergologie, Universitätsklinikum Ulm; ${ }^{59}$ Hautklinik, Universität Erlangen-Nürnberg, Erlangen; ${ }^{60}$ Fachklinik Hornheide, Münster; ${ }^{61}$ Klinik für Dermatologie, Venerologie und Allergologie, Universitätsklinikum, Kiel; ${ }^{62}$ Fachklinik für Dermatologie, Vitalklinik, Alzenau; ${ }^{63}$ Klinik für Dermatologie, Klinikum Lippe, Detmold; ${ }^{64}$ Klinik für Dermatologie, Allergologie und Umweltmedizin, Klinikum Schwabing, München; ${ }^{65}$ Universitäts-Hautklinik, Eberhards-Karls-Universität Tübingen; ${ }^{66}$ Fachklinikum, Borkum; ${ }^{67}$ Hautlinik, Klinikum Hildesheim; ${ }^{68}$ Klinik für Dermatologie und Allergologie, Harzklinikum Dorothea Christiane Erxleben, Quedlinburg; ${ }^{69} \mathrm{Klinik}$ für Dermatologie, Venerologie und Allergologie, Universitätsklinikum des Saarlandes, Homburg/Saar; ${ }^{70}$ Hautklinik, Klinikum Bremerhaven; ${ }^{71}$ Klinik für Dermatologie, Klinikum Augsburg; ${ }^{72}$ Dermatologie, DRK-Nordsee-Reha-Klinik Goldene Schlüssel, Sankt Peter-Ording; ${ }^{73}$ Klinik für Dermatologie und Allergologie, Krankenhaus Dresden-Friedrichstadt, Dresden;

${ }^{74}$ Klinik für Dermatologie, Allergologie und Venerologie, Universitätsklinikum Schleswig-Holstein, Lübeck;

${ }^{75}$ Klinik für Dermatologie, Venerologie und Allergologie/Immunologisches Zentrum, Städtisches Klinik Dessau, Dessau; ${ }^{76}$ Klinik für Dermatologie, Venerologie und Allergologie, Charite, Berlin; ${ }^{77}$ Klinik für Dermatologie und Allergologie, Klinikum Bremen-Mitte, Bremen daraus 320 Mio. EUR für die Gesamtwirtschaft $[1,2]$. Diese Kosten waren höher als die Summe, die zur Behandlung arterieller Erkrankungen ausgegeben wurde [10]. In Deutschland waren im Jahr 2006 0,3 von 1000 weiblichen und 0,5 von 1000 männlichen Versicherten der größten deutschen Krankenversicherung (AOK) aufgrund eines Ulcus cruris arbeitsunfähig [13].

\section{Phlebologie in der Dermatologie}

Die Therapie von Venenleiden findet neben der hausärztlichen Versorgung bei Fachärzten für Dermatologie, Gefäßchirurgie oder Allgemeinchirurgie statt. Auf der Basis der Facharztbezeichnungen Allgemeinmedizin, Chirurgie, Gefäßchirurgie und Dermatologie ist es möglich, die Zusatzbezeichnung Phlebologie zu erwerben.

In der Vergangenheit hatte die Diagnostik und Behandlung von Venenerkrankungen einen hohen Stellenwert in der Dermatologie. Die Phlebologie besitzt auch weiterhin einen festen Platz in der Weiterbildungsordnung der Dermatologie.

Dennoch zeigte sich in den letzten Jahren der dermatologische Anteil der Mitglieder der Deutschen Gesellschaft für Phlebologie sinkend, der Anteil der Allgemeinmediziner blieb konstant, während die Chirurgen und Gefäßchirurgen anteilig zulegten (Jahresbericht der Deutschen Gesellschaft für Phlebologie 2011, - Abb. 1). Allerdings liegt der Anteil der Dermatologen unter den Mitgliedern der DGP immer noch bei ca. 35\% (333 von 953, Stand 2011).

Aus diesen Ergebnissen resultierten Fragen:

- Welchen Stellenwert hat die Phlebologie aktuell an den dermatologischen Ausbildungsstätten junger Assistenzund Fachärzte?

- Welchen Stellenwert hat die Phlebologie aktuell in der Dermatologie?

- Wie umfangreich ist das aktuell an den dermatologischen Kliniken angebotene Spektrum der phlebologischen Diagnostik und Therapie? 


\section{Material und Methoden}

Zur Beantwortung dieser Fragen initiierten wir im Auftrag der Deutschen Gesellschaft für Phlebologie eine Umfrage an allen Hautkliniken in Deutschland. Eingeschlossen wurden alle Typen von Kliniken mit dermatologischen Abteilungen wie Universitätskliniken, nichtuniversitäre Akutkliniken, Rehakliniken und Belegabteilungen. Insgesamt wurden im $\mathrm{Au}$ gust 2012120 Kliniken (teils Doppelspitzen, 126 Klinikleiter) angeschrieben und um Beantwortung eines einseitigen Fragebogens gebeten (• Abb. 2). Der Fragebogen enthielt eine Vierteilung in

- Weiterbildung und Kooperation,

- Diagnostik,

- Therapie und

- Maßnahmen beim Ulcus cruris venosum als schwerste Form der chronischen venösen Insuffizienz.

Die Antworten sollten via Fax zunächst bis zum 01.10.2012 an die angegebene Nummer zurückgesandt werden. Bei initial nur mäßigem Rücklauf wurde ein Erinnerungsschreiben mit verlängertem Rücksendeintervall verschickt.

\section{Ergebnisse}

Von den initial insgesamt 120 angeschriebenen Kliniken erwiesen sich 3 als nicht mehr postalisch erreichbar. Die Zahl der auswertbaren Kliniken reduzierte sich somit auf 117. Von diesen erhielten wir bis zum 03.12.2012 76 auswertbare Fragebögen $(100 \%, n=76)$ zurück. (Ein Fragebogen wurde ohne Absender oder Faxsendenummer geschickt und konnte daher nicht in die Auswertung eingeschlossen werden.) Dies entspricht einer Rücklaufquote von $65 \%$.

Dabei machten die Akutkliniken den höchsten Anteil aus (Unikliniken 40,8\%, Akutkliniken 48,7\%, Rehakliniken 5,31\%, Belegabteilungen 2,6\%). Von den 76 Antwortenden wurde an nur 4 Kliniken keine phlebologische Diagnostik und Therapie durchgeführt.

S. Reich-Schupke · J. Alm · P. Altmeyer · D. Bachter · C. Bayerl · S. Beissert · T. Bieber · J. Böhmer · D. Dill · E. Dippel · P. Dücker · I. Effendy · S. El Gammal · P. Elsner · A. Enk · I. Feldmann-Böddeker · H. Frank · W. Gehring · U. Gieler · M. Goebeler · T. Görge · H. Gollnick · S. Grabbe · G. Gross · W. Gudat · A. Happ · R. Herbst · B. Hermes · N.-P. Hoff · S.M. John · M. Jungelhülsing · M. Jünger · M. Kaatz · A. Kapp · R. Kaufmann · J. Klode · K. Knaber · A. König · T. Krieg · P. Kohl · L. Kowalzick • P. Lehmann · H. Löffler · J. Maschke · W. Marsch · D. Mechtel · P. Mohr · I. Moll · M. Müller · D. Nashan · H.M. Ockenfels · R.U. Peter · H. Pillekamp · R. Rompel · T. Ruzicka · K. Salfeld · C. Sander · J. Schaller · K. Scharffetter-Kochanek · G. Schuler · H.-J. Schulze · T. Schwarz · B. Splieth · H. Stege • W. Stolz · A. Strölin · H. Tran · M. Tronnier · J. Ulrich · T. Vogt · G. Wagner · J. Welzel · T. Willgeroth · U. Wollina $\cdot$ D. Zillikens $\cdot$ C.C. Zouboulis $\cdot$ T. Zuberbier $\cdot$ M. Zutt $\cdot$ M. Stücker

\section{Phlebologie an deutschen Hautkliniken. Eine Bestandsaufnahme im Auftrag der Deutschen Gesellschaft für Phlebologie}

\section{Zusammenfassung}

Hintergrund. Venenleiden sind eine Volkskrankheit und haben eine enorme sozioökonomische Bedeutung. Dennoch scheint der Anteil der phlebologisch tätigen Dermatologen entsprechend den Zahlen der Deutschen Gesellschaft für Phlebologie (DGP) abzunehmen. Methoden. Um die Ursachen dieser Entwicklung zu untersuchen, führten wir im Herbst 2012 im Auftrag der DGP eine Befragungsstudie zur Phlebologie an 120 deutschen Hautkliniken durch.

Ergebnisse. In 76 antwortenden Klinien gab es im Mittel 1,5 Phlebologen und 0,9 Ärzte mit Voraussetzungen zur Weiterbildungsermächtigung Phlebologie. In 71,1\% der Kliniken gab es einen Phlebologen, in 73,7\% eine phlebologische Sprechstunde. Zur Diagnostik wurden besonders die Doppler- (89,5\%) und Duplexsonographie $(86,8 \%)$ eingesetzt. In der Therapie führte die Kompressionstherapie (94,7\%),

\section{Phlebology in German departments of dermatology. An analysis on behalf of the German Society of Phlebology}

\section{Abstract}

Background. Phlebologic diseases have become extremely common and have major socio-economic impact. However, the percentage of dermatologists working in phlebology appears to be decreasing according to the data of the German Society of Phlebology (DGP).

Methods. To investigate the reasons for this development, we - on behalf of the DGPsent a questionnaire to 120 German Departments of Dermatology in autumn 2012. Results. In 76 returned questionnaires, the number of physicians with additional fellowship training in phlebology averaged 1.5; the average number of those who fulfill the criteria for training fellows in phlebology was 0.9 . In $71.1 \%$ of the departments there was a phlebologist. A special phlebologic outpatient clinic existed in $73.7 \%$ of the departments. Sonography with Doppler (89.5\%) and duplex (86.8\%) was used as the most fre- gefolgt von Sklerosierungstherapien (flüssig $78,9 \%$, Schaum 63,2\%, Katheter 18,4\%), den endoluminalen thermischen Verfahren (Radiowelle $28,9 \%$, Laser $17,1 \%$ ) und den operativen Eingriffen (vor allem Cross- und Saphenektomie $67,1 \%$, Seitenastexhairese $75 \%$ ). Die durchschnittlichen Behandlungszahlen waren sehr heterogen.

Schlussfolgerung. Die Phlebologie spielt in der Dermatologie weiterhin eine wichtige Rolle. Mehrheitlich sind in den Kliniken die Möglichkeiten zur Weiterbildung Phlebologie gegeben. Es wird ein breites Spektrum an phlebologischen Techniken zur Diagnostik und Therapie angeboten.

Schlüsselwörter

Varikose · Chronische venöse Insuffizienz . Weiterbildung · Venenleiden · Dermatologen quent diagnostic tool. For therapy, compression $(94.7 \%)$, sclerotherapy (liquid $78.9 \%$, foam $63.2 \%$, catheter $18.4 \%$ ), endoluminal thermic procedures (radio wave $28.9 \%$, laser $17.1 \%$ ) and surgery (especially crossectomy and stripping $67.1 \%$, phlebectomy of tributaries $75 \%$ ) were used. The average number of treatments was very heterogenous in the different departments.

Conclusions. Phlebology definitely plays an important role in dermatology. Most departments fulfill the formal criteria for the license to conduct advanced training in phlebology. A wide spectrum of phlebological diagnostic and therapeutic procedures is available.

\section{Keywords} Varicose veins - Chronic venous insufficiency . Additional fellowship training · Phlebology . Dermatologists 


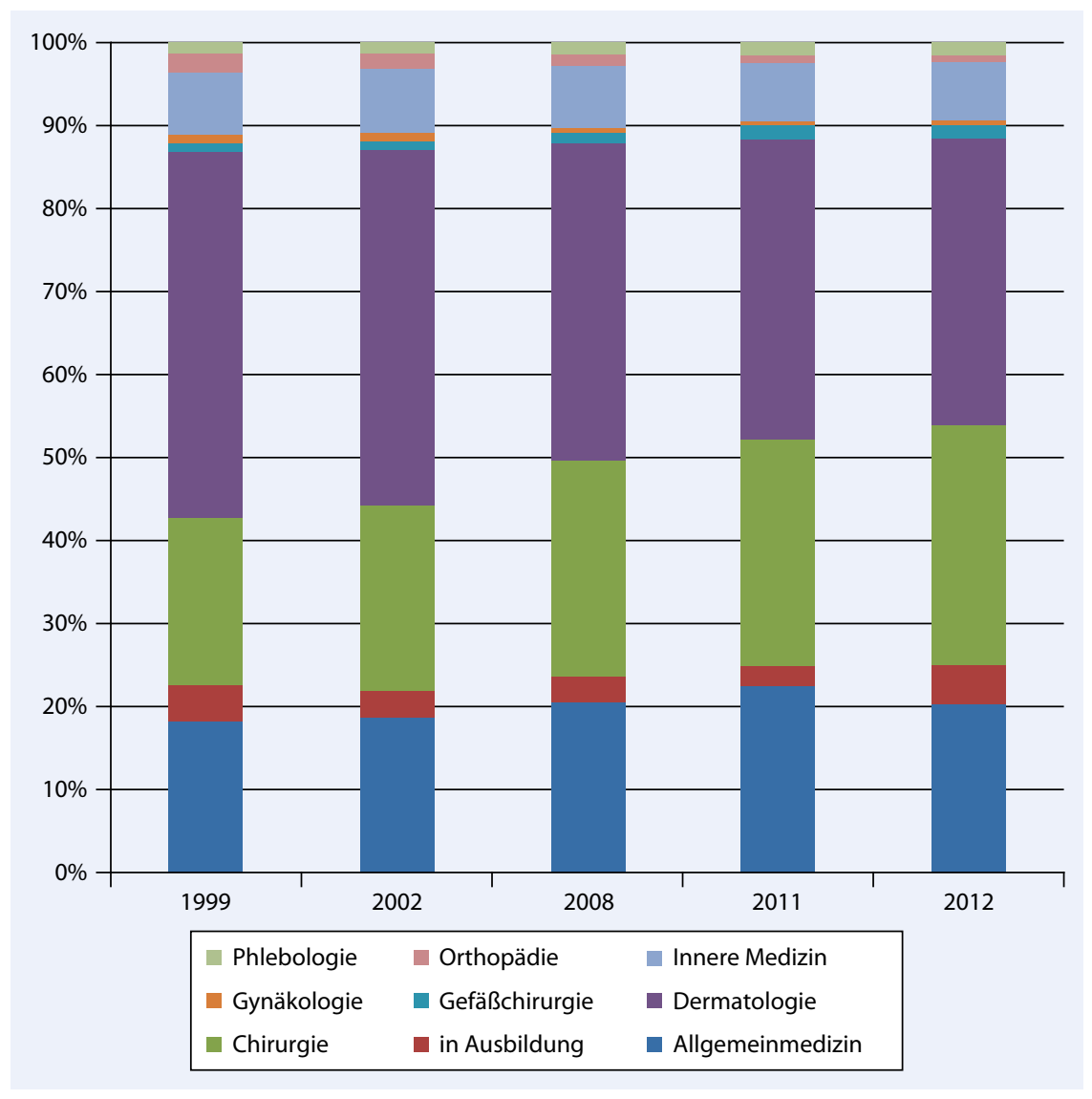

Abb. $1 \Delta$ Mitgliederzusammensetzung der Deutschen Gesellschaft für Phlebologie nach Fachgebiet im zeitlichen Verlauf der letzten 13 Jahre

\section{Zusatzbezeichnung und Weiterbildungsermächtigung}

Die Anzahl der Ärzte mit der Zusatzbezeichnung Phlebologie an den Kliniken liegt im Mittel bei 1,51 ( $\pm 1,75$, Median 1,0$)$. Im Mittel gibt es an den Kliniken 0,90 $( \pm 1,20$, Median 1,0$)$ Ärzte, die die Voraussetzungen für die Weiterbildungsermächtigung Phlebologie erfüllen. Dabei schwankte die Zahl der Ärzte pro Klinik stark (• Abb. 3, 4). In 71,1\% der Kliniken gibt es mindestens einen Arzt mit phlebologischer Zusatzbezeichnung. In immerhin 20,9\% der Kliniken gibt es mehr als 2 Phlebologen. Die Voraussetzungen für die Weiterbildungsermächtigung Phlebologie erfüllen in 39,5\% der Kliniken 1 Arzt, in nur 3,9\% der Kliniken mehr als 2 Ärzte. Insgesamt besteht in 76,3\% der Kliniken eine Weiterbildungsmöglichkeit.

Entsprechend den teils additiv eingegangenen schriftlichen Rückmeldungen zu den ausgefüllten Fragebögen gibt es jedoch in zahlreichen Kliniken Bestrebun-

\section{Phlebologische Diagnostik}

Im Rahmen der phlebologischen Diagnostik werden an den Kliniken digitale Photoplethysmographie (dPPG; 56,6\%), Venenverschlussplethysmographie (VVP; 42,1\%), Dopplersonographie (89,5\%), Duplexsonographie $(86,8 \%)$, Phlebographie (52,6\%), Ovarikographie (14,5\%) und Angio-MRT $(57,9 \%)$ eingesetzt. Die Zahlen der pro Monat durchgeführten Untersuchungen schwanken in den Kliniken stark (• Tab. 1).

\section{Phlebologische Therapie}

Das Spektrum der therapeutischen Optionen umfasst konservative, interventionelle und operative Methoden. Angewendet werden in den antwortenden Kliniken Kompressionstherapien (94,7\%), Flüssigsklerosierungen $(78,9 \%)$, Schaumsklerosierungen $(63,2 \%)$, Kathetersklerosierungen $(18,4 \%)$, Clarivein $(5,3 \%)$, endoluminale Radiowellentherapie $(28,9 \%)$ und die endoluminale Lasertherapie (17,1\%). Bei den operativen Optionen werden extraluminale Valvuloplastien (5,3\%), Crossund Saphenektomien $(67,1 \%)$, Seitenastexhairesen $(75,0 \%)$ und Recrossektomien $(48,7 \%)$ genannt. Auch hier schwanken die Anzahlen der durchgeführten Therapiemaßnahmen zwischen den einzelnen Kliniken extrem (• Tab. 2).

\section{Maßnahmen bei Patienten mit Ulcus cruris venosum}

In den 76 Kliniken mit phlebologischer Diagnostik und Therapie gibt es in 73,7\% der Kliniken eine eigene phlebologische Sprechstunde mit durchschnittlich 103 ( $\pm 198,1$ Patienten, Median 22,5) Patienten pro Monat; 84,2\% der Kliniken geben eine Kooperation mit anderen Abteilungen zur Versorgung phlebologischer Patienten an, in $47,4 \%$ der Fälle über ein Konsilwesen, in $35,5 \%$ über Zentrenbildung ( $n=13 \mathrm{Kli}$ niken keine Antwort zu dieser Frage). Die häufigsten kooperierenden Fachgruppen sind in diesem Zusammenhang allein oder in weiterer Kooperation Gefäßchirurgie (68,5\%), Angiologie (44,8\%), Kardiologie (25,0\%) und Radiologie (2,6\%).

In den antwortenden Kliniken erhalten Patienten mit einem Ulcus cruris eine Gefäßdiagnostik $(94,7 \%)$, einen bakteriologischen Abstrich (96,1\%), eine Kompressionstherapie (94,7\%), eine Sanierung der Gefäßsituation (77,6\%), eine Spalthauttransplantation $(93,4 \%)$ und eine intermittierende Unterdrucktherapie (85,5\%). Auch hier war die Bandbreite der pro Monat durchgeführten Behandlungen erheblich (• Tab. 3).

\section{Diskussion}

\section{Weiterbildung Phlebologie}

Die dargestellten Zahlen zeigen, dass die Phlebologie innerhalb der Dermatologie 


\section{Fragebogen zur Bestandserhebung von Diagnostik und Therapie chronischer venöser Insuffizienz (CVI) in Deutschland}

1. Betreiben Sie in Ihrer Klinik Diagnostik und Therapie der CVI?

- $\quad$ nein $\rightarrow$ Ende des Fragebogens.

- ja $\rightarrow$ Bitte weiter mit Frage 2.

2. Bieten Sie in Ihrer Ambulanz eine spezielle phlebologische Sprechstunde an?

- O nein $\mathrm{O}$ ja $\rightarrow$ wenn ja, wie viele Patienten pro Monat?

3. Wie viele Ärzte in Ihrer Klinik tragen die Zusatzweiterbildung Phlebologie?

4. Wie viele Ärzte in Ihrer Klinik erfüllen die Kriterien zur Weiterbildungsermächtigung Phlebologie?

5. Besteht in Ihrem Hause eine Kooperation mit einer anderen Fachdisziplin bzgl. der Diagnostik und Therapie von CVI?

nein

ja O Im Rahmen einer Konsiltätigkeit $\quad \mathrm{O}$ im Rahmen einer gemeinsamen Zentrenbildung

Welche anderen Fachrichtungen sind an der Zentrenbildung beteiligt?

O Gefäßchirurgie O Angiologie O Kardiologie $O$ andere:

6. Welche Verfahren zur Diagnostik von CVI nutzen Sie in Ihrer Klinik?

- Venenverschlussplethysmographie

Dopplersonographie

$\mathrm{O}$ nein $\mathrm{O} \mathrm{ja} \rightarrow$ wenn $\mathrm{ja}$, wie häufig pro Monat?

- Duplexsonographie

$\mathrm{O}$ nein $\mathrm{O} \mathrm{ja} \rightarrow$ wenn $\mathrm{ja}$, wie häufig pro Monat?

- Phlebographie

$\mathrm{O}$ nein $\mathrm{O} \mathrm{ja} \rightarrow$ wenn $\mathrm{ja}$, wie häufig pro Monat?

$\mathrm{O}$ nein $\mathrm{O} \mathrm{ja} \rightarrow$ wenn $\mathrm{ja}$, wie häufig pro Monat?

Ovaricographie

$\mathrm{O}$ nein $\mathrm{O} j \mathrm{ja} \rightarrow$ wenn $\mathrm{ja}$, wie hăufig pro Monat?

$\mathrm{O}$ nein $\mathrm{O} \mathrm{ja} \rightarrow$ wenn $\mathrm{ja}$, wie häufig pro Monat?

- Angio-MRT

$\mathrm{O}$ nein $\mathrm{O} \mathrm{ja} \rightarrow$ wenn $\mathrm{ja}$, wie häufig pro Monat?

7. Welche Verfahren zur Therapie von CVI nutzen Sie in Ihrer Klinik?

\begin{tabular}{|c|c|c|}
\hline Kompressionstherapie & O nein & $\mathrm{O}$ ja $\rightarrow$ wenn ja, wie häufig pro Monat? \\
\hline Sklerosierungstherapie, flüssig & O nein & $\mathrm{O}$ ja $\rightarrow$ wenn ja, wie häufig pro Monat? \\
\hline Sklerosierungstherapie, Schaum & O nein & $\mathrm{O}$ ja $\rightarrow$ wenn ja, wie häufig pro Monat? \\
\hline \multicolumn{2}{|c|}{ Katheterassistierte Sklerosierungstherapie } & $\mathrm{O}$ nein $\mathrm{O}$ ja $\rightarrow$ wenn $\mathrm{ja}$, wie häufig pro \\
\hline Clarivein & O nein & $\mathrm{O}$ ja $\rightarrow$ wenn ja, wie häufig pro Monat? \\
\hline Endoluminale Radiowellentherapie & O nein & $\mathrm{O}$ ja $\rightarrow$ wenn ja, wie häufig pro Monat? \\
\hline Endoluminale Lasertherapie & O nein & $\mathrm{O}$ ja $\rightarrow$ wenn ja, wie häufig pro Monat \\
\hline Extraluminale Valvuloplastie & O nein & O ja $\rightarrow$ wenn ja, wie häufig pro Monat? \\
\hline Cross- und Saphenektomie & O nein & $\mathrm{O}$ ja $\rightarrow$ wenn ja, wie häufig pro Monat? \\
\hline Seitenastexhairese/ Phlebektomie & O nein & $\mathrm{O}$ ja $\rightarrow$ wenn ja, wie häufig pro Monat? \\
\hline Re-Crossektomie & O nein & $\mathrm{O}$ ja $\rightarrow$ wenn ja, wie häufig pro Monat? \\
\hline
\end{tabular}

8. Welche Maßnahmen führen Sie in Ihrer Klinik beim Ulcus cruris durch?

\begin{tabular}{lll} 
- & Gefäßdiagnostik (aUSD + Duplexsonographie) & $\mathrm{O}$ nein $\mathrm{O} j \mathrm{ja} \rightarrow$ wenn ja, wie häufig pro Monat? \\
- & Bakteriologischer Abstrich & $\mathrm{O}$ nein $\mathrm{O} j \mathrm{ja} \rightarrow$ wenn ja, wie häufig pro Monat? \\
- & Kompressionstherapie & $\mathrm{O}$ nein $\mathrm{O} j \mathrm{ja} \rightarrow$ wenn ja, wie häufig pro Monat? \\
- & Sanierung der Gefäßsituation & $\mathrm{O}$ nein $\mathrm{O} \mathrm{ja} \rightarrow$ wenn ja, wie häufig pro Monat? \\
- & Spalthauttransplantation & $\mathrm{O}$ nein $\mathrm{O} j \mathrm{ja} \rightarrow$ wenn ja, wie häufig pro Monat? \\
- & Sog-Therapie (z.B. VAC) & $\mathrm{O}$ nein $\mathrm{O} j \mathrm{ja} \rightarrow$ wenn ja, wie häufig pro Monat? \\
\hline
\end{tabular}

Bitte via Fax an PD Dr. Stefanie Reich-Schupke 0234-8792-376. DANKE!

Abb. $2<$ Verwendeter Fragebogen einen sehr heterogenen Stellenwert einnimmt. Einserseits gibt es starke phlebologische Zentren mit einer großen Zahl an Phlebologen und Weiterbildungsbefugten, andererseits gibt es aber auch einen Teil dermatologischer Kliniken (23,7\%), an denen zwar phlebologische Diagnostik und Therapien durchgeführt werden, die aber keine phlebologische Weiterbildungsstätte mehr darstellen. Gleichzeitig gibt es entsprechend der uns mit den Fragebögen zugesandten Begleitschreiben of- fenbar erhebliche Bemühungen, die Phlebologie in der Dermatologie zu behalten und in den Kliniken mit derzeit schwächerer phlebologischer Situation wieder auszubauen. Diese Bestrebungen sind letztlich vor allem auch vor dem Hinter- 

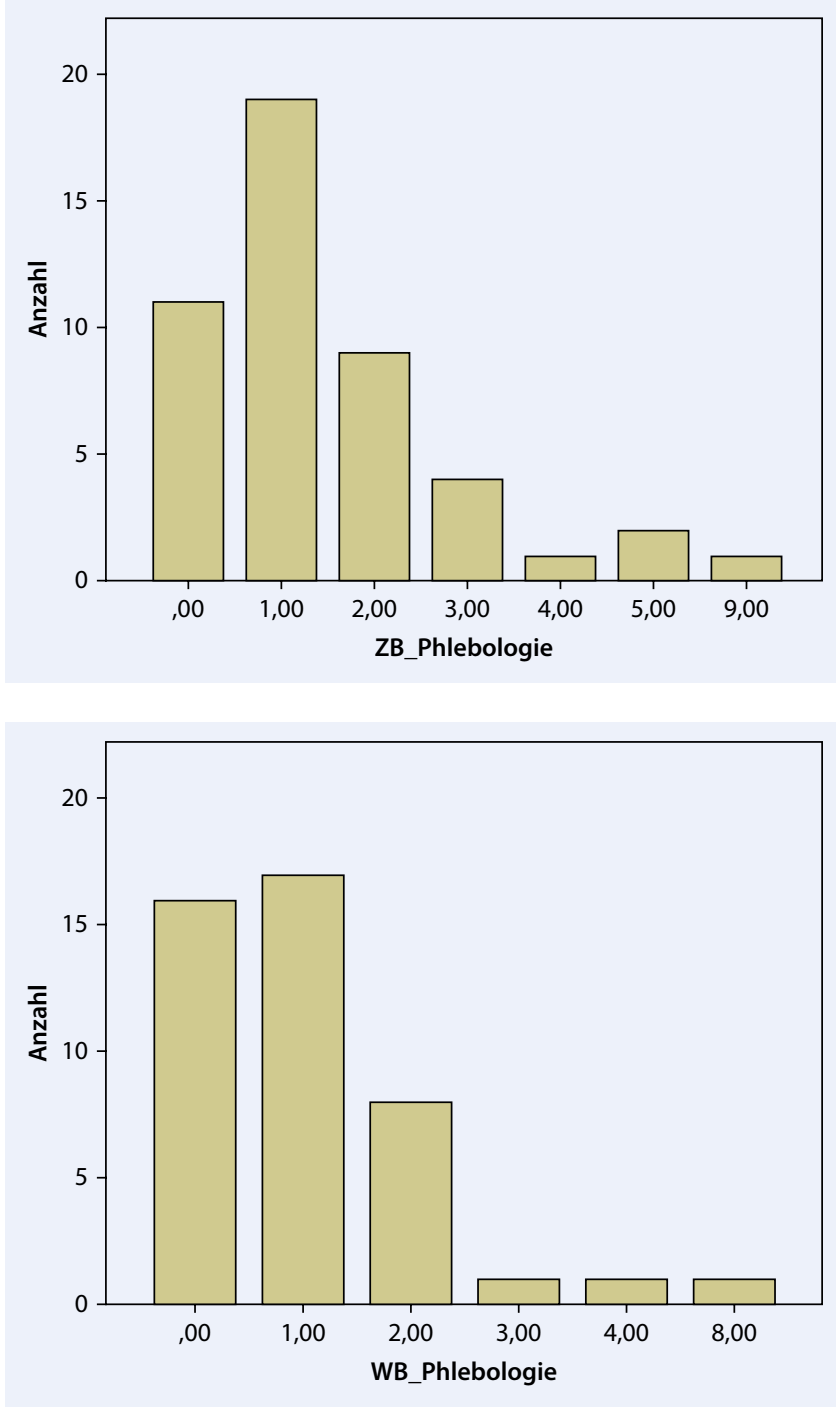

\section{Phlebologische Publikationen}

Auch in dermatologischen Fachzeitschriften hat die Phlebologie weiterhin einen festen Platz. Nimmt man beispielhaft für die Dermatologie im deutschsprachigen Raum das Journal der Deutschen Dermatologischen Gesellschaft (JDDG) aus dem Jahr 2011, so waren immerhin 6,5\% der insgesamt 139 erschienenen Originalarbeiten, Reviews, Kasuistiken und Letter aus dem phlebologischen Gebiet. Thematisch beschäftigten sich immerhin 3 Beiträge mit dem Ulcus cruris, 5 mit der venösen Insuffizienz (4 davon allerdings waren Reaktionen auf dieselbe Originalarbeit) sowie einer mit dem Lipödem. Wenngleich die Auswertung eines Journals aus einem Jahr natürlich keine repräsentativen Zahlen bietet, so zeigen die Daten doch, dass die Phlebologie auch wissenschaftlich weiterhin einen Platz in der Dermatologie innehat.

\section{Phlebologische Diagnostik und Therapie erfolgen weitgehend ambulant}

Die Phlebologie in Deutschland findet in weiten Teilen im niedergelassenen Bereich statt. Das in den Praxen angebotene Spektrum umfasst nicht überall die komplette phlebologische Diagnostik und Therapie. Vielfach besteht daher nur eine Weiterbildungsermächtigung für wenige Monate. Es ist somit sowohl für die Praxisinhaber als auch für die Ärzte, die eine Weiterbildungsstätte suchen, umso schwerer, eine komplette phlebologische Weiterbildung zu erhalten. Eigentlich ist dies nur in Schwerpunktzentren möglich.

\section{Phlebologische Fortbildung ohne Möglichkeit des Erwerbs der Zusatzbezeichnung Phlebologie}

Dennoch gibt es eine ganze Reihe von phlebologisch Interessierten mit vielfach breitem phlebologischem Wissen, denen es jedoch aus organisatorischen Gründen nicht möglich ist, eine phlebologische $\mathrm{Zu}-$ satzbezeichnung zu erlangen. Dieser Entwicklung trägt die Deutsche Gesellschaft für Phlebologie Rechnung und hat eine Fortbildungsakademie gegründet, durch die ein phlebologisches Fortbildungszer- 


\begin{tabular}{|lllll}
\hline $\begin{array}{l}\text { Tab. } 1 \text { Häufigkeit der verschiedenen Diagnostikmethoden pro Monat in den antwortenden } \\
\text { Kliniken }\end{array}$ & $\begin{array}{l}\text { Anzahl der Klini- } \\
\text { ken mit Zahlen- } \\
\text { angaben }\end{array}$ & $\begin{array}{l}\text { Mittel Patien- } \\
\text { ten/Monat }\end{array}$ & Range & Median \\
\hline Diagnostikmethode & 81,1 & $0-1000$ & 15,0 \\
\hline $\begin{array}{l}\text { Digitale Photoplethysmo- } \\
\text { graphie }\end{array}$ & 36 & 49,3 & $0-1000$ & 5,0 \\
\hline $\begin{array}{l}\text { Venenverschlussplethys- } \\
\text { mographie }\end{array}$ & 30 & 86,0 & $0-1000$ & 30,0 \\
\hline Dopplersonographie & 61 & 86,5 & $0-850$ & 25,0 \\
\hline Duplexsonographie & 66 & 2,6 & $0-20$ & 2,0 \\
\hline Phlebographie & 29 & 0,3 & $0-2$ & 0,0 \\
\hline Ovarikographie & 14 & 4,7 & $0-20$ & 2,0 \\
\hline Angio-MRT & 37 & & & \\
\hline
\end{tabular}

Tab. 2 Häufigkeit der verschiedenen Therapiemethoden pro Monat in den antwortenden Kliniken

\begin{tabular}{|lllll}
\hline Therapiemethode & $\begin{array}{l}\text { Anzahl der Kliniken mit } \\
\text { Zahlenangaben }\end{array}$ & $\begin{array}{l}\text { Mittel Patien- } \\
\text { ten/Monat }\end{array}$ & Range & Median \\
\hline Kompression & 59 & 99,7 & $0-850$ & 50,0 \\
\hline Sklerotherapie flüssig & 52 & 28,3 & $0-500$ & 10,0 \\
\hline Sklerotherapie Schaum & 43 & 28,5 & $0-500$ & 10,0 \\
\hline Kathetersklerotherapie & 10 & 0,2 & $0-2$ & 0,0 \\
\hline Clarivein & 13 & 0,4 & $0-3$ & 0,0 \\
\hline $\begin{array}{l}\text { Endoluminale Radiowellen- } \\
\text { therapie }\end{array}$ & 21 & 8,0 & $0-80$ & 2,0 \\
\hline $\begin{array}{l}\text { Endoluminale Laser- } \\
\text { therapie }\end{array}$ & 19 & 4,2 & $0-40$ & 0,0 \\
\hline $\begin{array}{l}\text { Extraluminale Valvulo- } \\
\text { plastie }\end{array}$ & 14 & 3,3 & $0-20$ & 0,0 \\
\hline Cross- und Saphenektomie & 47 & 19,9 & $0-380$ & 5,0 \\
\hline Seitenastexhairese & 50 & 21,7 & $0-250$ & 5,0 \\
\hline Recrossektomie & 36 & 12,2 & $0-200$ & 1,5 \\
\hline
\end{tabular}

Tab. 3 Maßnahmen bei Patienten mit Ulcus cruris venosum pro Monat in den antwortenden Kliniken

\begin{tabular}{|lllll}
\hline Maßnahme & $\begin{array}{l}\text { Anzahl der } \\
\text { Kliniken mit } \\
\text { Zahlenangaben }\end{array}$ & $\begin{array}{l}\text { Mittel Patien- } \\
\text { ten/Monat }\end{array}$ & Range & Median \\
\hline Gefäßdiagnostik & 64 & 26,3 & $0-200$ & 20,0 \\
\hline Bakteriologischer Abstrich & 61 & 50,9 & $0-250$ & 30,0 \\
\hline Kompressionstherapie & 60 & 69,0 & $0-600$ & 47,5 \\
\hline Sanierung der Gefäßsituation & 49 & 11,3 & $0-50$ & 10,0 \\
\hline Spalthauttransplantation & 62 & 8,9 & $0-150^{\text {a }}$ & 5,0 \\
\hline $\begin{array}{l}\text { Intermittierende Unterdruck- } \\
\text { therapie }\end{array}$ & 60 & 9,3 & $0-200^{\text {a }}$ & 4,0 \\
\hline aHier eine Klinik als Ausreißer nach oben im Vergleich zu anderen Kliniken. & & \\
\hline
\end{tabular}

tifikat erworben werden kann. Diese ermöglicht phlebologisch interessierten Kollegen eine entsprechende phlebologische Weiterbildung mit abschließender Zertifizierung durch die DGP, auch wenn dies nicht zum Erwerb der Zusatzbezeichnung Phlebologie führt. Weiterhin bietet die DGP für ihre Mitglieder ein Hospitations-

\section{Phlebologische Diagnostik}

Als Goldstandard in der phlebologischen Diagnostik gilt sowohl bei der Varikose, der Thrombose als auch beim Ulcus cruris die Duplexsonographie. Die Umsetzung dieser Empfehlungen spiegelt sich auch in den angegebenen Zahlen der Kliniken wider. Hier sind die Ultraschalldoppler- und Duplexsonographie die Diagnostikmethode mit dem höchsten Patientenaufkommen pro Monat (• Tab. 1). Bildgebende Diagnostik mit Einsatz von Kontrastmitteln macht nur eine kleine Zahl von wenigen Patienten im Monat in den antwortenden Einrichtungen aus. Eine Indikation zur Phlebographie besteht angesichts der außerordentlich hoch auflösenden sonographischen Verfahren nur noch in seltenen Fällen bei duplexsonographisch schwer einzuordnenden Befunden, pathologischen Veränderungen in der Beckenetage oder bei Verdacht auf kongenitale Anomalien. Die Ovarikographie bleibt den seltenen Fällen bei klinischem Verdacht auf das Vorliegen eines pelvinen Stauungssyndroms vorbehalten. Rezidivierende Oberschenkelvarizen, zyklusabhängige Schmerzen und Stauungsbeschwerden in den Beinen, genitale Varizen sowie Schmerzen beim Geschlechtsverkehr können hier hinweisend sein [7].

Neben der reinen Funktionskontrolle der Venen ist die Duplexsonographie vor allem wichtig für die Planung des therapeutischen Procedere [8]. Die Bildgebung ermöglicht hier zusätzlich zu den Refluxsignalen eine Bestandserhebung zur Morphologie und Lagezuordnung der verschiedenen Venenabschnitte.

\section{Phlebologische Therapie}

Das therapeutische Spektrum der Varikose ist derzeit so breit wie nie zuvor $[6,8]$. Es reicht von konservativen Maßnahmen (Kompressionstherapie) über die Sklerosierungstherapie, endoluminale Katheterverfahren bis hin zur operativen Intervention mit verschiedenen Techniken. Im Vergleich der angegebenen Zahlen stellt die Sklerosierungstherapie (Flüssigkeit und Schaum) mit einem Mittelwert von ca. 60 Patienten pro Monat neben der operativen Intervention (verschiedene Verfahren) mit ca. 57 Patienten pro Monat 
die wichtigste Therapieoption der antwortenden Kliniken dar ( $\bullet$ Tab. 2). Die vielfach stark umworbenen und bezüglich ihrer Langzeitergebnisse diskutierten endoluminalen Verfahren werden im Mittel nur bei ca. 13 Patienten pro Monat eingesetzt. Insgesamt stehen somit in den antwortenden Kliniken konservative Maßnahmen klar im Vordergrund.

Besonders bei Betrachtung der angegebenen Maßnahmen zur Therapie des Ulcus cruris als schwerster Form der chronischen venösen Insuffizienz scheint in den meisten Kliniken die Kompressionstherapie als symptomatisch orientierte, konservative Maßnahme eindeutig im Vordergrund zu stehen. Im Vergleich dazu haben leider die kausal orientierte Diagnostik (Gefäßdiagnostik) und die kausale Therapie der Varikose (Sanierung der Gefäßsituation) einen geringeren Stellenwert. Diese Divergenz könnte unter Berücksichtigung der Empfehlungen der aktuellen Leitlinien zum Ulcus cruris und der aktuellen S3-Leitlinie der Lokaltherapie chronischer Wunden zur kausal orientierten Therapie noch weiter vermindert werden $[3,6,8]$.

\section{Fazit für die Praxis}

\section{- Zusammenfassend zeigen die Daten der Selbstauskünfte der Kliniken, dass die Phlebologie weiter eine wichtige Rolle in der Dermatologie spielt. \\ - An immerhin ca. zwei Drittel der ant- wortenden Hautkliniken besteht die formale Weiterbildungsberechtigung Phlebologie. \\ - Davon unabhängig werden phlebo- logische Diagnostik, konservative, in- terventionelle und operative Thera- pieoptionen von einem wesentlichen Anteil der Hautkliniken allein oder in Kooperationsmodellen ausgeübt.}

\section{Korrespondenzadresse}

\section{PD Dr. S. Reich-Schupke}

Klinik für Dermatologie, Venerologie und Allergologie, Venenzentrum der Dermatologischen und Gefäßchirurgischen Kliniken, Ruhr-Universität Bochum Hiltroper Landwehr 11-13, 44805 Bochum stefanie.reich-schupke@rub.de

\section{Einhaltung ethischer Richtlinien}

Interessenkonflikt. S. Reich-Schupke, J. Alm, P. Altmeyer, D. Bachter, C. Bayerl, S. Beissert, T. Bieber, J. Böhmer, D. Dill, E. Dippel, P. Dücker, I. Effendy, S. El Gammal, P. Elsner, A. Enk, I. Feldmann-Böddeker, H. Frank, W. Gehring, U. Gieler, M. Goebeler, T. Görge, H. Gollnick, S. Grabbe, G. Gross, W. Gudat, A. Happ, R. Herbst, B. Hermes, N.-P. Hoff, S.M. John, M. Jungelhülsing, M. Jünger, M. Kaatz, A. Kapp, R. Kaufmann, J. Klode, K. Knaber, A. König, T. Krieg, P. Kohl, L. Kowalzick, P. Lehmann, H. Löffler, J. Maschke, W. Marsch, D. Mechtel, P. Mohr, I. Moll, M. Müller, D. Nashan, H.M. Ockenfels, R.U. Peter, H. Pillekamp, R. Rompel, T. Ruzicka, K. Salfeld, C. Sander, J. Schaller, K. Scharffetter-Kochanek, G. Schuler, H.-J. Schulze, T. Schwarz, B. Splieth, H. Stege, W. Stolz, A. Strölin, H. Tran, M. Tronnier, J. Ulrich, T. Vogt, G. Wagner, J. Welzel, T. Willgeroth, U. Wollina, D. Zillikens, C.C. Zouboulis, T. Zuberbier, M. Zutt und M. Stücker geben an, dass kein Interessenkonflikt besteht.

Dieser Beitrag beinhaltet keine Studien an Menschen oder Tieren.

\section{Literatur}

1. Allaert FA, Causse C (2002) Pharmaco-epidemiology of the treatment of chronic venous insufficiency in general medicine. Int Angiol 21 (2 Suppl 1):1217

2. Allaert FA, Cazaubon M, Causse C et al (2005) Venous disease and ergonomics of female employment. Int Angiol 24:265-271

3. Burckhardt M, Gregor S, Kleijnen J et al (2012) Leitlinienreport: Lokaltherapie chronischer Wunden bei Patienten mit den Risiken periphere arterielle Verschlusskrankheit, Diabetes mellitus, chronische venöse Insuffizienz. Leitlinienreport 091-001

4. Deutsche Gesellschaft für Phlebologie (2008) Leitlinie zur Therapie und Diagnostik des Ulcus cruris venosum (8/2008). Phlebologie 37:308-329

5. Dinkel R (1997) Venous disorders, a cost intensive disease. Phlebology 26:164-168

6. Gloviczki P, Gloviczki ML (2012) Guidelines for the management of varicose veins. Phlebology 27(Suppl 1):2-9

7. Kies DD, Kim HS (2012) Pelvic congestion syndrome: a review of current diagnostic and minimally invasive treatment modalities. Phlebology 27(Suppl 1):52-57

8. Kluess HG, Noppeney T, Breu FX et al (2010) Leitlinie zur Diagnostik und Therapie des Krampfaderleidens. Phlebologie 39:271-289

9. McGuckin M, Waterman R, Brooks J et al (2002) Validation of venous leg ulcer guidelines in the United States and United Kingdom. Am J Surg 183:132-137

10. Nicolaides A, Allegra C, Bergan JJ et al (2008) Management of chronic venous disorders of the lower limbs. Guidelines according to scientific evidence. Int Angiol 27:1-59

11. Purwins S, Herberger K, Debus ES et al (2010) Costof-illness of chronic leg ulcers in Germany. Int Wound J 7:97-102

12. Rabe E, Pannier-Fischer F, Bromen K et al (2003) Bonner Venenstudie der Deutschen Gesellschaft für Phlebologie. Epidemiologische Untersuchung zur Frage der Häufigkeit und Ausprägung von chronischen Venenkrankheiten in der städtischen und ländlichen Wohnbevölkerung. Phlebologie 32:1-14
13. Rabe E, Bauersachs R, Pannier F, List SM (2009) Gesundheitsberichterstattung des Bundes 2009. Venenerkrankungen der Beine, Heft 44. Robert-KochInstitut, Berlin

14. Rabe E, Pannier F (2010) Societal costs of chronic venous disease in CEAP C4, C5, C6 disease. Phlebology 25(Suppl 1):64-67 